\title{
The site of a missense mutation in the extracellular Ig or FN domains of L1CAM influences infant mortality and the severity of $\mathrm{X}$ linked hydrocephalus
}

\author{
Ron C Michaelis, Yang-Zhu Du, Charles E Schwartz
}

\begin{abstract}
The $L 1$ cell adhesion molecule (L1CAM) plays an important role in axon growth, fasciculation, and neural migration. Mutations in the L1CAM gene produce a phenotype characterised by $X$ linked hydrocephalus, mental retardation, spastic paraplegia, adducted thumbs, and agenesis of the corpus callosum. We have conducted a detailed analysis of the phenotypic effects of missense mutations in the extracellular portion of L1CAM, following a study that differentiated between "key" amino acid residues critical for maintaining the conformation of the extracellular immunoglobulin type C-like (Ig) or fibronectin type III-like (FN) domains and surface residues of less certain significance. We have analysed the data from 71 published cases and seven patients whose mutations were detected in our laboratory to determine if the site of a missense mutation in the Ig or FN domains correlated with the severity of hydrocephalus, presence of adducted thumbs, or survival past infancy. Mutations affecting the key residues in either type of domain were more likely to produce a phenotype with severe hydrocephalus, adducted thumbs, and lifespan less than one year than were mutations affecting surface residues. In addition, mutations affecting the FN domains were more likely than those affecting Ig domains to produce a phenotype with severe hydrocephalus, with less certain effects on adducted thumbs and lifespan. Mutations in key residues of the FN domains were particularly deleterious to infant survival. These data provide information that may be useful in predicting some aspects of the phenotypic effects of certain L1CAM mutations.

(FMed Genet 1998;35:901-904)
\end{abstract}

Keywords: L1CAM; cell adhesion molecules; missense mutations; genotype-phenotype

The $\mathrm{L} 1$ cell adhesion molecule (L1CAM) is a $200 \mathrm{kDa}$ cell surface glycoprotein involved in cell-cell adhesion, neuronal cell migration, neurite outgrowth and fasciculation, and myelination..$^{1-5}$ Mutations in the L1CAM gene produce a phenotype in which hydrocephalus, mental retardation, spastic paraplegia, ad- ducted thumbs, and agenesis of the corpus callosum are the most consistent features. ${ }^{6-10}$ The L1CAM phenotype is particularly noteworthy for its variability. Not only do mutations in a particular domain of the protein produce highly variable effects, but families have been reported in which different members carrying the same mutation manifested different combinations of phenotypic features. ${ }^{11-13}$ As with any disorder, establishing correlations between the site of mutations and the severity or specific features of the resultant phenotype will provide useful information for families in which L1CAM mutations are found. Previous attempts to establish genotype-phenotype correlations for L1CAM mutations have separated L1CAM mutations into classes along rather broad lines. ${ }^{7-10}$ The present study represents an attempt to use previously reported information about the amino acid structure of the extracellular domains of the L1CAM protein to conduct a more detailed analysis of the phenotypic effects of missense mutations in the extracellular portion of the L1CAM gene.

The extracellular portion of the L1CAM protein contains six immunoglobulin C2-like (Ig) domains and five fibronectin type III-like (FN) domains. This is followed by a transmembrane segment and a cytoplasmic region thought to be associated with several protein kinases. ${ }^{14}{ }^{15}$ In the first 80 families reported to carry L1CAM mutations, 75 different mutations were reported, with only three mutations appearing in multiple families. ${ }^{16}$ These mutations have been distributed throughout all portions of the LICAM gene, and include deletions as well as frameshift, nonsense, missense, and splice site mutations. ${ }^{17-22}$ The original consensus that there was little correlation between mutation site and phenotype came from the general observation that different patients with mutations affecting the same domain of the L1CAM protein frequently had phenotypes that differed in their severity or the appearance of specific features. ${ }^{11-13}$ More recent and systematic attempts to establish genotypephenotype correlations have divided LICAM mutations into groups along very broad lines. One study has reported that mutations that either truncate or grossly rearrange the structure of the L1CAM protein have more severe phenotypic effects than missense mutations that result in a single amino acid substitution. ${ }^{7}$ Another study has measured the severity of hydrocephalus, the presence/absence of adducted thumbs, and the incidence of death 
Table 1 Phenotypic features of the seven patients whose mutations were analysed in our laboratory

\begin{tabular}{llll}
\hline $\begin{array}{l}\text { Mutation site } \\
(A A)\end{array}$ & Hydrocephalus & $\begin{array}{l}\text { Adducted } \\
\text { thumbs }\end{array}$ & $\begin{array}{l}\text { Survival } \\
\text { past 1 } y\end{array}$ \\
\hline S674C & Severe & - & Yes \\
A691D & Severe & - & No \\
& Severe & Yes & - \\
G698R & Severe & - & No \\
& Severe & - & No \\
G698R & Severe & - & No \\
\hline
\end{tabular}

Patients are grouped according to family. Data not available are indicated by -

before 1 year of age, and classified L1CAM mutations into three classes of increasing severity: mutations affecting the cytoplasmic domain, missense mutations in the extracellular domain, and mutations truncating the protein in the extracellular domain. ${ }^{10}$

Mutations truncating the protein in the extracellular domain should leave the LICAM molecule without a means by which to attach to the cell membrane. Such a mutation would be expected to produce a complete loss of function, and the severe phenotype that results from these truncating mutations is consistent with this prediction. Similarly, the finding that mutations that grossly rearrange the structure of the L1CAM protein exert more severe phenotypic effects than mutations causing a single amino acid substitution is also consistent with our understanding of structure-function relationships. While these studies do provide information that can be useful in predicting the severity of the phenotype resulting from truncations or gross rearrangements of the L1CAM protein, these findings do not help predict the phenotypic effects of the many L1CAM mutations that involve missense mutations in the extracellular domains. Bateman $e t a l^{3}$ have recently provided a means by which missense mutations in the extracellular domains of L1CAM can be classified according to their function. These authors have differentiated between the amino acid residues necessary for maintaining conformation of the Ig and FN domains (key residues) of LICAM and those on the surface of their respective domains. We have applied this information in an effort to increase the ability to predict the severity of the phenotype resulting from specific missense mutations in the extracellular domains of L1CAM. We have analysed the severity of hydrocephalus, the presence/ absence of adducted thumbs, and survival beyond one year in 71 published patients and seven patients whose mutations were detected in our laboratory, all of whom had missense mutations in the extracellular Ig or FN domains of L1CAM.

\section{Subjects and methods}

Informed consent was obtained from all seven subjects whose mutations were analysed in our laboratory before obtaining samples for mutation analysis. This study was approved by the Institutional Review Board of Self Memorial Hospital, Greenwood, SC.

Descriptions of the mutation status and phenotypic features of the 71 patients reported previously can be found as follows: 52 patients are described in the table describing class 2 mutations in the report by Yamasaki et $\mathrm{al}^{1 \mathrm{0}} ; 13^{\mathrm{C}}$ patients are described in the L1 Mutation Web Page (Van Camp et al, ${ }^{16}$ Web Nos 21, 30, 33, $35,41,45,52,61,63,66,67,69,70$ ); four $\mathbb{\Phi}$ patients (HC25, HC5, HC29, HC11) areథ

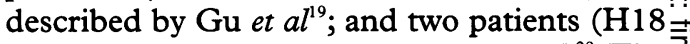
and $\mathrm{H}_{12}$ ) are described by Jouet et al. ${ }^{20}$ The $\stackrel{\text { की }}{?}$ phenotypic features of the seven patients whose? mutations were analysed in our laboratory are $\frac{\overline{\underline{T}}}{\overline{\underline{n}}}$ listed in table 1. The mutations have been $\frac{\bar{\sigma}}{\bar{\sigma}}$ reported for five of these seven patients, two with A691D and three with G698R, by Du etes $a l^{17}$

Hydrocephalus was scored as severe if it was accompanied by aqueductal stenosis, if the $\vec{\rho}$ patient required a ventriculoperitoneal shunt, if the hydrocephalus was marked enough to beo diagnosed in utero, or if the hydrocephalus was ${ }_{\tilde{J}}^{\omega}$ described as progressive. Hydrocephalus involving ventricular enlargement without mac-ie rocephaly and not requiring shunting was clas- -9 sified as moderate. The presence versus absence of adducted thumbs was observed, as was whether the patient survived longer than $Z$ one year after birth. Data regarding thumbs and survival were not available for all patients. In some cases, the patient was within a year@ after birth at the time of writing. Data were $\overrightarrow{0}$ analysed using the chi square test. For $2 \times 2 \%$ analyses, the Yates correction factor was used. ${ }^{24}$

\section{EXON NUMBERING}

The L1CAM gene has historically been considered to have 28 exons, with the ATG codon signalling the beginning of translation in exon 1. It has recently been discovered, however, that there is an additional $13 \mathrm{~kb}$ of genomic DNA at the $5^{\prime}$ end of the L1CAM gene. ${ }^{25}$ The actual exon 1 is approximately 10. $\mathrm{kb}$ upstream from what has historically been considered exon 1 , and the actual exon 2 is $183^{\circ}$ bp long, composed of the $76 \mathrm{bp}$ that have his 3 . torically been considered exon 1 plus an additional 107 bases 5 ' to the original ATG. The $₹$ exon numbering system used in this papero reflects the new exon numbering system. How- $\rightarrow$ ever, there are no data presented for analysis of any of the newly discovered 5' regions. Analysesn for exon 2 refer not to the entire exon, but only to the $169 \mathrm{bp}$ that constitute what has historically been considered exon 1 plus 43 and $50 \mathrm{bpc}$ of flanking sequence on the $5^{\prime}$ and $3^{\prime}$ sides, respectively. According to the new nomencla- $-\frac{\mathrm{O}}{\tau}$ ture, this includes the most 3' $119 \mathrm{bp}$ of exon $2_{\mathscr{N}}^{\Phi}$ plus the most 5' 50 bp of intron 2. Protocols are currently being developed to analyse the newly discovered 5' regions of L1CAM for mutations. The results of these analyses will be reported separately.

SINGLE STRAND CONFORMATION POLYMORPHISM (SSCP)

Exons 2, 3, 4, 5, 20, 26, 27+28, and 29 wereo analysed by SSCP. The respective exon and some flanking intronic sequence were amplified by polymerase chain reaction (PCR) using standard protocols and primer sequences reported earlier. ${ }^{20}$ The electrophoretic 
Table 2 Hydrocephalus, adducted thumbs, and death during the first year after birth in patients with missense mutations in the extracellular FN and Ig domains of L1CAM

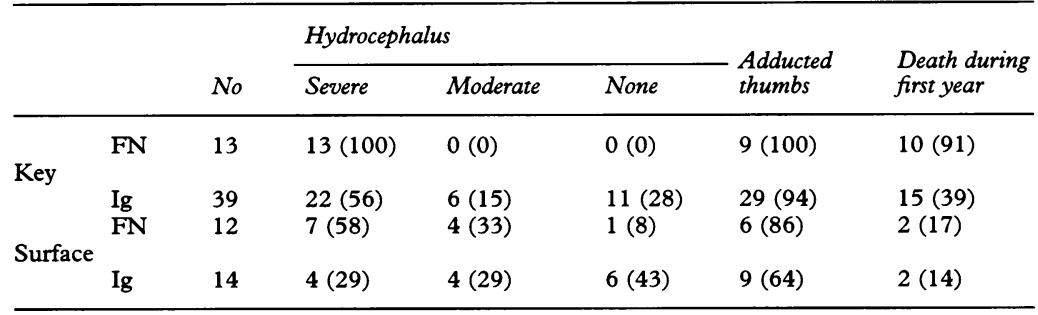

Entries represent the number (percentage) of patients with the specified mutation site showing the respective feature.

mobilities of the PCR products from control and patient samples were compared using either manual SSCP gels or the Automated Laser Fluorescence (ALF) system (Pharmacia Biotech). For manual analysis, PCR products were $5^{\prime}$ end labelled using polynucleotide kinase and $\gamma$-P32 ATP, and electrophoresed through $0.5 \times \mathrm{MDE} / 1 \times \mathrm{TBE}$ gels at room temperature, using $8 \mathrm{~W}$ power, for 14 hours. Fragments were visualised by autoradiography at room temperature. For automated analyses, samples were electrophoresed through a $0.5 \times$ $\mathrm{MDE} / 5 \%$ glycerol $/ 1.2 \times \mathrm{TBE}$ gel, with $50 \mathrm{~W}$ power. To maximise detection, SSCP was performed on all samples at $14^{\circ} \mathrm{C}$ and $30^{\circ} \mathrm{C}$, and on some others at $20^{\circ} \mathrm{C}$ as well. Any PCR products showing abnormal migration were regenerated and directly sequenced.

\section{RESTRICTION ENDONUCLEASE FINGERPRINTING (REF)}

Exons 6+7, 8-11, 12-16, 17-19, and 21-25 were analysed using REF. In L1CAM, these exons cluster closely enough that each exon group, along with some of its surrounding intronic sequences, can be amplified in individual PCRs. Restriction enzyme digests, end labelling, and gel electrophoresis were performed as described elsewhere. ${ }^{17} 18$

PCR CYCLE SEQUENCING

Abnormally migrating PCR products (SSCP) or restriction fragments (REF) were regenerated by PCR, and purified by a spin column method (Qia-Quick, Qiagen). Purified templates were sequenced using the Promega fmol cycle sequencing kit with $\alpha-{ }^{32} \mathrm{P}-\mathrm{dCTP}$. Products were electrophoresed through $6 \%$ acrylamide $/ 7 \mathrm{~mol} / 1$ urea gels, and autoradiography was performed at room temperature.

\section{Results}

KEY VERSUS SURFACE RESIDUES

Table 2 illustrates the phenotypic differences resulting from mutations in the different domains. While the percentage of patients with some degree of hydrocephalus in each group was similar, mutations in key residues tended to produce more severe hydrocephalus than mutations in surface residues $\left(\chi^{2}(2)=5.44\right.$, $\mathrm{p}<0.10)$. Adducted thumbs were more common in patients with mutations in key residues than surface residues $\left(\chi^{2}(1)=4.94, p<0.05\right)$. In addition, patients with mutations in key residues were more likely to die within the first year of life than patients with mutations in surface residues $\left(\chi^{2}(1)=7.6, p<0.01\right)$.
FN VERSUS IG DOMAINS

Mutations in the FN domains were more likely than mutations in the Ig domains to produce hydrocephalus, especially severe hydrocephalus $\left(\chi^{2}(2)=8.7, p<0.02\right)$. In contrast, there was no significant difference between $\mathrm{FN}$ and Ig mutations with respect to the presence of adducted thumbs or the percentage of patients who died within the first year of life.

\section{KEY FN RESIDUE MUTATIONS ARE ESPECIALLY} DELETERIOUS TO SURVIVAL

There was a marked difference in the effect that key versus surface mutations exerted on survival depending on whether the mutations were in $\mathrm{FN}$ or $\mathrm{Ig}$ domains $\left(\chi^{2}(3)=18.5\right.$, $\mathrm{p}<0.01)$. For the Ig domains, survival rates were relatively high for patients with mutations in either key or surface residues $\left(\chi^{2}(1)=1.9\right.$, NS). However, for the FN domains, mutations in key residues had a significantly more deleterious effect on survival than did mutations in surface residues $\left(\chi^{2}(1)=9.8, p<0.01\right)$. No such interaction was seen with respect to the severity of hydrocephalus or the presence of adducted thumbs.

\section{Discussion}

The fact that mutations in key residues of L1CAM carry more severe phenotypic consequences than mutations in surface residues is predictable given our understanding of the importance of protein conformation to protein function. There is too little known regarding the relative importance of the extracellular Ig and FN domains of L1CAM to brain development, however, to make a similar judgement regarding the greater incidence of hydrocephalus seen in patients with mutations in the FN versus Ig domains. The specific roles played by the Ig and FN domains may be cell specific. It has been suggested that the second Ig domain is involved in the homophilic binding that promotes neurite outgrowth from retinal cells. ${ }^{26} 27$ In contrast, all six Ig domains and the first two FN domains have been shown to promote neurite outgrowth from small cerebellar neurones. $^{28}$

The fact that $\mathrm{FN}$ domain mutations are more likely to produce hydrocephalus than Ig domain mutations may reflect either an important ligand binding function of the FN domain or the relative positions of these domains on the L1CAM molecule. Mutations in key residues will no doubt disrupt ligand binding in the specific domain where the mutation occurs. However, mutations in the key residues of the FN domains could also exert indirect effects on Ig domain function, by either inducing a conformational change in the LICAM protein that leaves the Ig domains less than maximally accessible to their ligands, or by interfering with signal transduction normally initiated by ligand binding at the Ig domains.

Consistent with previous reports, most patients with L1CAM mutations, regardless of the mutation site, had adducted thumbs, ${ }^{6}$ while more variability was seen with respect to the severity of hydrocephalus and survival past infancy. Our findings suggest that mutations in 
key residues can be expected to produce more severe hydrocephalus and poorer odds for survival than mutations in surface residues. In addition, mutations in the extracellular FN domains can be expected to produce more severe hydrocephalus, and to a lesser extent poorer chances for survival past infancy, than mutations in the Ig domains. Finally, mutations in key residues of the extracellular FN domains are particularly deleterious to survival. There is still a lot that is unclear regarding the genotype-phenotype relationships for L1CAM mutations. However, separating amino acids from the extracellular Ig and $\mathrm{FN}$ domains on the basis of their role as key versus surface amino acids may help predict some aspects of the phenotype that results from a missense mutation in one of the L1CAM extracellular domains.

1 Asou H, Miura M, Kobayashi M, Uyemura K, Itoh K. Cell adhesion molecule $\mathrm{L} 1$ guides cell migration in primary reaggregation cultures of mouse cerebellar cells. Neurosci Lett 1992;144:221-4.

2 Lemmon V, Farr K, Lagenaur C. L1-mediated axon outgrowth occurs via a homophilic binding mechanism. Neuron 1989;2:1597-603.

3 Miura M, Asou H, Kobayashi M, Uyemura K. Functional expression of a full-length cDNA coding for rat neural cell adhesion molecule $\mathrm{L} 1$ mediates homophilic intercellular adhesion and migration of cerebellar neurons. $\mathcal{F}$ Biol Chem adhesion and migratic

4 Rathjen FG, Wolff JM, Frank R, Bonhoeffer F, Rutishause U. Membrane glycoproteins involved in neurite fasciculation. $\mathcal{F}$ Cell Biol 1987; 104:343-53.

5 Wood PM, Schachner M, Bunge RP. Inhibition of Schwann cell myelination in vitro by antibody of the $\mathrm{Ll}$ adhesion molecule. $\mathcal{F}$ Neurosci 1990;10:3635-45.

6 Fransen E, Lemmon V, Van Camp G, Vits L, Caucke P, Willems PJ. CRASH syndrome: clinical spectrum of corpus callosum hypoplasia, retardation, adducted thumbs, spastic callosum hypoplasia, retardation, adducted thumbs, spastic paraparesis and hydrocephalus due to mutation

single gene, L1. Eur f Hum Genet 1995;3:273-84.
7 Fransen D, Vits L, Van Camp G, Willems PJ. The clinical spectrum of mutations in $\mathrm{L} 1$ a neuronal cell adhension molecule. Am ₹ Med Genet 1996;64:73-7.

8 Kenwrick S, Jouet M, Donnai D. X linked hydrocephalus and MASA syndrome. 7 Med Genet 1996;33:59-65.

9 Ruiz JC, Cuppens H, Legius E, et al. Mutations in L1CAM in two families with $\mathrm{X}$ linked complicated spastic paraplegia, MASA syndrome, and HSAS. $₹$ Med Genet 1995;32. 549-52.

10 Yamasaki M, Thompson P, Lemmon V. CRASH syndrome: mutations in L1CAM correlate with severity of the disease. Neuropediatrics 1997;28:175-8.
11 Fransen E, Schrander-Stumpel C, Vits L, Coucke P, Van Camp G, Willems P. X-linked hydrocephalus and MASA syndrome present in one family are due to a single missensec mutation in exon 28 of the LiCAM gene. Hum Mol Genet 1994;3:225-6.

12 Fried K. X-linked mental retardation and/or hydrocephalus. Clin Genet 1972;3:258-63.

13 Willems PJ, Brouwer OF, Dijsktra I, Wilmink J. X-linked hydrocephalus. Am 7 Med Genlet 1987;27:921-8.

14 Wong EV, Schaefer AW, Landreth G, Lemmon V. Involve-: ment of $\mathrm{p} 90^{\text {rs }}$ in neurite outgrowth mediated by the cell adhesion molecule L1*. F Biol Chem 1996;271:18217-23. ज

15 Wong EV, Schaefer AW Landreth G, Lemmon V. Caseino kinase II phosphorylates the neural cell adhesion molecule L1. F Neurochem 1996;66:779-86.

16 Van Camp G, Fransen E, Vits L, Raes G, Willems PJ. A locus-specific mutation database for the neural cellథ adhesion molecule L1CAM (Xq28). Hum Mutat 1996;8: $\frac{\text { ? }}{391-6 .}$
3um

17 Du YZ, Srivastava AK, Schwartz CE. Multiple exon screening using restriction endonulease fingerprinting (REF): $\overrightarrow{0}$ detection of six novel mutations in the Ll cell adhesionmolecule (L1CAM) gene. Hum Mutat (in press).

18 Du YZ, Dickerson C, Aylsworth AS, Schwartz CE. AO "silent" mutation, C924T (G308G), in the L1CAM generesults in X linked hydrocephalus (HSAS). 7 Med Genet 1998;35:456-62.

19 Gu SM, Orth U, Veske A, et al. Five novel mutations in the L1CAM gene in families with $\mathrm{X}$ linked hydrocephalus. $\mathcal{F}$ Med Genet 1996;33:103-6.

20 Jouet M, Rosenthal A, Armstrong G, et al. X-linked spastic paraplegia (SPG1), MASA syndrome and X-linked hydrocephalus result from mutations in the $\mathrm{Ll}$ gene. Nature $\mathrm{O}$ 1995;7:402-7.

21 MacFarlane JR, Du JS, Pepys ME, et al. Nine novel L1CAMmutations in families with X-linked hydrocephalus. Hum Mutat 1997;9:512-18.

22 Takechi $\mathrm{T}$, Tohyama J, Kurachige $\mathrm{T}$, et al. A deletion of five $\Phi$ nucleotides in the LICAM gene in a Japanese family with X-linked hydrocephalus. Hum Genet 1996;97:353-6.

23 Bateman A, Jouet M, MacFarlane J, Du JS, Kenwrick, S, Chothia C. Outline structure of the human L1 celladhesion molecule and the sites where mutations cause neurological disorders. EMBO f 1996;15:6050-9.

24 Strickberger MW. Genetics. New York: MacMillan, 1968: 135.

25 Kallunki P, Edelman GM, Jones FS. Tissue-specific expression of the L1 cell adhesion molecule is modulated by the neural restrictive silencer element. F Cell Biol 1997;138: 1343-54

26 Zhao X, Siu CH. Colocalization of the homophilic binding site and the neuritogenic activity of the cell adhesion molecule L1 to its second Ig-like domain. $\mathcal{f}$ Biol Chem $\overrightarrow{\bar{O}}$ 1995;270:29413-21.

27 Zhao X, Siu CH. Differential effects of two hydrocephalus/ MASA syndrome-related mutations on the homophilic binding and neuritogenic activities of the cell adhesion molecule L1. F Biol Chem 1996;271:6563-6.

28 Appel F, Holm J, Conscience JF, Schachner M. Several extracellular domains of the neural cell adhesion molecule? L1 are involved in neurite outgrowth and cell body adhesion. $\mathcal{F}$ Neurosci 1993;11:4764-75. 